\title{
Tolerability and safety of real-world use of pomalidomide in patients with relapsed/refractory multiple myeloma
}

\author{
EISEKI USAMI $^{1}$, MICHIO KIMURA ${ }^{1}$, SHOYA TAKENAKA $^{1}$, MINA IWAI $^{1}$, \\ HITOMI TERAMACHI $^{2}$ and TOMOAKI YOSHIMURA ${ }^{1}$ \\ ${ }^{1}$ Department of Pharmacy, Ogaki Municipal Hospital, Ogaki, Gifu 503-8502; ${ }^{2}$ Department of Clinical Pharmacy, \\ Gifu Pharmaceutical University, Gifu, Gifu 501-1196, Japan
}

Received July 30, 2018; Accepted November 21, 2018

DOI: $10.3892 / \mathrm{mco} .2018 .1775$

\begin{abstract}
Pomalidomide (POM) is a second-generation immunomodulatory agent with proven efficacy in patients with relapsed/refractory multiple myeloma (RRMM) proven to be refractory to previous treatment with lenalidomide (LEN) and bortezomib. We herein conducted a retrospective analysis of 14 RRMM patients receiving POM to determine its tolerability and safety in the clinical setting. The median age of the patients was 72 years (range, 58-84 years), and $85.7 \%$ of the patients were aged $>70$ years. The most frequent treatment dose was $3 \mathrm{mg} / \mathrm{day}$. POM dose reductions were required in $54.5 \%(6 / 11)$ of the patients. The patient data were compared among three age groups $(<70,70-75$ and $>75$ years $)$ and there was only significant difference in daily POM treatment dose. The tolerability of POM must be confirmed, particularly in elderly patients. Dose reduction from 4 to $3 \mathrm{mg}$ occurred during the second cycle in $83.3 \%(5 / 6)$ of the patients. It is important to determine the tolerability of POM in the early phases of treatment. The most frequently reported grade $3 / 4$ hematological adverse events were neutropenia (64.3\%), anemia (64.3\%) and thrombocytopenia (57.1\%). Although the median number of treatment cycles was 4 (range, 1-13), 21.4\% (3/14) of the patients with a performance status (PS) of 3 were administered only 1 treatment cycle. The tolerability of POM was low among patients with poor PS and an aggressive treatment introduction should be avoided. However, $21.4 \%(3 / 14)$ of the patients were able to continue treatment for $>1$ year and some patients received long-term therapy. POM does not require dose modification for renal function, and multiple capsule doses are available, which is an advantage of POM compared with LEN. POM may be administered to late-stage RRMM patients in a real-world
\end{abstract}

Correspondence to: Mr. Eiseki Usami, Department of Pharmacy, Ogaki Municipal Hospital, 4-86 Minaminokawa-cho, Ogaki, Gifu 503-8502, Japan

E-mail: omhp2002@yahoo.co.jp

Key words: pomalidomide, tolerability, real-world use, relapsed/refractory multiple myeloma clinical setting, but elderly patients or those with poor PS must be treated with caution. In this manner, the treatment options for RRMM patients may be expanded by assessing the tolerability and safety of POM.

\section{Introduction}

Multiple myeloma (MM) is an incurable hematological malignancy caused by pathological proliferation of plasma cells. MM is a disease of older adults, with a median age at diagnosis of 66 years (1). The median survival of patients with MM has notably improved, from 3-4 years to $\sim 7-8$ years, due to the development of various new agents, such as immunomodulatory drugs (IMiDs), including thalidomide or lenalidomide (LEN), and proteasome inhibitors (PIs), such as bortezomib (BOR) $(2,3)$. Patients who relapse after or become refractory to IMiDs and BOR have a very poor prognosis, with a median overall survival (OS) of 9 months, or only 3 months if no further treatment is administered (4). Recently, survival has been further prolonged with the introduction of new PIs (carfilzomib or ixazomib), histone deacetylase inhibitors (panobinostat) and monoclonal antibodies (elotuzumab or daratumumab) (5).

Pomalidomide (POM) is a second-generation IMiD with different antitumor mechanisms compared with those of LEN (6). IMiDs can be administered per os, which is convenient and may particularly benefit older patients. The evidence regarding POM efficacy in patients with relapsed/refractory MM (RRMM) is based on a phase 3 trial (MM-003) that compared POM plus low-dose dexamethasone (DEX) therapy to high-dose DEX alone (7). Based on the results of the phase 2 trial (MM-002) (8) and MM-003, POM was approved in the United States, European Union and other countries in 2013. A recent phase $3 \mathrm{~b}$ trial (MM-010) proved the safety and efficacy of POM in a large population of RRMM patients refractory to LEN and BOR treatment $(9,10)$.

However, real-world data are scarce. POM has been approved only for patients refractory to LEN and BOR, and it may be administered to late-stage RRMM patients. Clinical trial data may not always reflect real-world clinical practice and outcomes. Thus, a retrospective analysis of RRMM patients receiving POM was conducted to determine its tolerability and safety in a real-world clinical setting. 


\section{Patients and methods}

Patients. The subjects of this study were RRMM patients who received POM at the Ogaki Municipal Hospital (Ogaki, Japan) between June 2015 and May 2018. POM was administered on days 1-21 of 28-day cycles with low-dose DEX on days 1,8 , 15 and 22 .

Patient background. The characteristics of the patients treated with POM were investigated to determine their sex, age, time from diagnosis, estimate glomerular filtration rate (eGFR) and laboratory data at initiation of POM treatment, myeloma subtype, cytogenetic abnormalities, international staging system categorization at diagnosis and prior treatment.

POM tolerability. The POM dosing information (exposure to POM, number of treatment cycles, daily treatment dose and initiation treatment dose) was examined. Patients were divided into three age groups ( $<70,70-75$ and $>75$ years), and laboratory data and POM treatment data were compared among these three groups.

POM safety. The adverse events (AEs) associated with POM treatment were recorded from electronic charts and medication management records. The reasons for POM dose reduction or interruption as a result of AEs were examined. The severity of AEs was classified according to the Common Terminology Criteria for Adverse Events, version 4.0 (11).

Statistical analysis. The data were analyzed using JMP software, version 5.0.1J (SAS Institute Japan Ltd., Tokyo, Japan). The Kruskal-Wallis test was used for comparisons among age groups. The recorded $\mathrm{P}$-values were two-sided and $\mathrm{P}<0.05$ was considered to indicate a statistically significant difference.

\section{Results}

Patient background. The baseline characteristics of 14 patients who received POM are listed in Table I. A total of 68 cycles were administered. The median age (range) of the patients was $72(58-84)$ years, and the patients received a median of $3(1-7)$ prior treatment regimens. A total of $85.7 \%(12 / 14)$ patients had been refractory to LEN and BOR treatment. Two patients had been treated with high-dose conventional therapy with autologous stem cell transplantation (HDT-ASCT), whereas the others were considered ineligible for HDT-ASCT.

POM tolerability. POM dosing information is shown in Table II. The median treatment duration and number of treatment cycles were 3.7 (0.9-14.3) months and 4 (1-13), respectively. A total of $21.4 \%(3 / 14)$ of the patients were able to continue treatment for $>1$ year. The median POM treatment dose was $3(1-4) \mathrm{mg} /$ day. The most frequent treatment doses were $3 \mathrm{mg} /$ day $(48.5 \%$ of the patients), and $4 \mathrm{mg} /$ day ( $41.2 \%$ of the patients). The initiation POM treatment dose was $4 \mathrm{mg}$ in $78.6 \%$ (11/14) of patients. Two patients were started on $3 \mathrm{mg}$ due to a history of low tolerability (hematological AEs) or ileus caused by LEN; the remaining patient was started on $2 \mathrm{mg}$ due to low tolerability (hematological AEs) caused by LEN. A comparison of laboratory data and POM treatment data among the three age categories $(<70$,
$70-75$ and $>75$ years) is presented in Table III. Although there was no significant difference in the baseline characteristics of the patients (performance status, prior treatment regimens, laboratory data or exposure to POM), there was a significant difference in the daily treatment dose $(\mathrm{P}<0.01)$.

POM safety. In the present study, the most frequently reported grade 3/4 hematological AEs were neutropenia (64.3\%), anemia (64.3\%) and thrombocytopenia (57.1\%). Infection was the most frequent grade 3/4 non-hematological AE (42.9\%), and fatigue occurred in $28.6 \%$ of the patients (Table IV). The reasons necessitating dose reduction or interruption of POM treatment are summarized in Table V. Dose reduction or interruption occurred in $50.0 \%$ of the patients, most commonly due to neutropenia (35.7\%) and thrombocytopenia (14.3\%). The rate of dose interruption due to fatigue was $21.4 \%$ (3/14). The rate of overall discontinuation due to AEs was 28.6\% (4/14).

\section{Discussion}

We herein describe the real-world use of POM administered to 14 RRMM patients at Ogaki Municipal Hospital. In the present study, all the patients were considered as ineligible for HDT-ASCT when they received treatment with POM. Prior treatment comprised $>3$ regimens in almost all patients. POM was used at late-stage RRMM. The median patient age (range) was 72 (58-84) years, which was higher compared with that reported in other real-world or large-scale phase 3 trial data 59 (32-78) years in Sriskandarajah et al (12), 61 (41-82) years in Maciocia et al (13) and 66 (37-88) years in the MM-010 trial (9). In the present study, $85.7 \%$ of patients were aged $>70$ years, which was a higher rate compared with the other studies. The patient characteristics were analyzed among the three age groups $(<70,70-75,>75$ years $)$. There was no difference in treatment initiation dose, exposure to POM or number of treatment cycles. Although the initiation treatment dose was $4 \mathrm{mg} / \mathrm{day}$ in $78.6 \%(11 / 14)$ of the patients, the most frequent treatment dose was $3 \mathrm{mg}$ /day ( $48.5 \%$ of total treatment courses). POM dose reductions were required in $54.5 \%(6 / 11)$ of the patients. The frequency of dose reduction was higher compared with that in the MM-010 (22.0\%) and MM-011 (25.0\%) trials. There was only a significant difference in daily treatment dose among age groups. The tolerability of POM must be particularly confirmed in elderly patients. In the present study, the reduction from 4 to $3 \mathrm{mg}$ occurred during the second treatment cycle in $83.3 \%(5 / 6)$ of the patients. It is important to recognize the tolerability of each patient to POM in the early stages. Ailawadhi et al (14) reported long durations of treatment and response, higher response rates and fewer AEs with $2 \mathrm{mg}$ POM. Therefore, the efficacy and safety of POM has been reported even at lower doses, and treatment dose must be reduced according to each patient's tolerability.

In the IFM2009-02 trial, 39.7\% of the patients were able to continue treatment for $>1$ year. There was a significant difference between the two groups ( $\geq 1$ vs. $<1$ year of treatment). The progression-free survival (PFS) was 20.7 vs. 4.6 months. Similarly, the OS rate was 100 vs. $66 \%$ at 12 months and 91 vs. $40 \%$ at 18 months, respectively (15). Sriskandarajah et al (12) reported a significant effect in 61.5\% (24/39) of patients who were able to continue treatment for $>5$ cycles. Long-term treatment 
Table I. Baseline characteristics of the patients.

\begin{tabular}{cc}
\hline No. (\%) or median \\
[range]
\end{tabular}

Number of patients

14

Sex

Male

Female

6

Age (years)

$\geq 70$

$>75$

Time from diagnosis (years)

ECOG performance status

$0-1$

2-3

eGFR at initiation of

pomalidomide treatment

$\left(\mathrm{ml} / \mathrm{min} / 1.73 \mathrm{~m}^{2}\right)$

eGFR $\geq 60$

$60>$ eGFR $\geq 30$

30> eGFR

Laboratory data at initiation

of pomalidomide treatment

WBC (per $\mu \mathrm{l})$

Neutrophils (per $\mu \mathrm{l})$

Hemoglobin $(\mathrm{g} / \mu \mathrm{l})$

Platelets (x10 $10^{4}$ per $\left.\mu \mathrm{l}\right)$

Myeloma subtype

IgG

IgA

Light chain only

Cytogenetic abnormalities del (17p)

$\mathrm{t}(4: 14)$

t $(14: 16)$

Other

International staging system at diagnosis

I

II

III

Prior treatment

Prior treatment regimens

$>2$ previous regimens

Dexamethasone

Lenalidomide

Bortezomib

Thalidomide

Carfilzomib

Ixazomib

Cyclophosphamide ${ }^{\mathrm{a}}$

Melphalan $^{\text {b }}$

Stem cell transplantation

2.2

9

5

$\begin{array}{ll}7 & \\ 4 & (50.0) \\ 3 & (28.6) \\ & (21.4)\end{array}$

(28.6)

3

$(0.0)$

$(0.0)$

$(0.0)$

(21.4)

3

6
Table I. Continued.

\begin{tabular}{|c|c|c|}
\hline Characteristics & \multicolumn{2}{|c|}{$\begin{array}{c}\text { No. }(\%) \text { or median } \\
\text { [range] }\end{array}$} \\
\hline \multicolumn{3}{|l|}{ Refractory to prior therapies } \\
\hline Lenalidomide & 13 & $(92.9)$ \\
\hline Bortezomib & 13 & $(92.9)$ \\
\hline Both lenalidomide and bortezomib & 12 & $(85.7)$ \\
\hline \multicolumn{3}{|c|}{$\begin{array}{l}\text { ancluding cyclophosphamide that was administered for stem cell } \\
\text { mobilization. 'Including high-dose melphalan. ECOG, Eastern } \\
\text { Cooperative Oncology Group; eGFR, estimated glomerular filtration } \\
\text { rate; WBC, white blood cell; Ig, immunoglobulin. Data are presented } \\
\text { as n }(\%) \text { or median [range]. }\end{array}$} \\
\hline
\end{tabular}

Table II. Pomalidomide dosing information.

[0.7-14.2]

$\begin{array}{cc}4,560 & {[2,300-8,380]} \\ 2,759 & {[1,035-7,039]} \\ 9.4 & {[6.9-11.9]} \\ 14.4 & {[6.1-29.9]}\end{array}$

continuity with POM has been shown to lead to prolonged PFS and OS. In the present study, only $21.4 \%$ of the patients were able to continue treatment for $>1$ year. Gueneau et al (16) reported POM effectiveness in the early recurrence phase. However, Palmas et al (17) reported its effectiveness in heavily pretreated patients. Therefore, the timing of POM treatment initiation must be individualized. Some patients were able to continue with long-term treatment, and POM may be expected to remain continuously effectiveness. However, 21.4\% (3/14) of the patients could only tolerate 1 treatment cycle. These patients were unable to receive the next treatment due to the deterioration of their PS. One patient had first been diagnosed 14 years prior and was heavily pretreated. The other patients were refractory to carfilzomib. The PS of all three patients was 3 when they received 
Table III. Comparison of laboratory data and pomalidomide treatment data between age categories.

\begin{tabular}{|c|c|c|c|c|}
\hline \multirow[b]{2}{*}{ Factors } & \multicolumn{3}{|c|}{ Age categories (years) } & \multirow[b]{2}{*}{ P-value } \\
\hline & $<70$ & $70-75$ & $>75$ & \\
\hline Number of patients & 2 & 7 & 5 & 0.39 \\
\hline ECOG performance status & $1[0-1]$ & $2[0-3]$ & $1[0-3]$ & 0.29 \\
\hline Time from diagnosis, median (years) & $4.0[1.6-6.3]$ & $1.7[0.7-14.2]$ & $3.2[0.8-9.3]$ & 0.85 \\
\hline Prior treatment regimens & $3[2-3]$ & $3[2-6]$ & $3[1-7]$ & 0.82 \\
\hline \multicolumn{5}{|l|}{$\begin{array}{l}\text { Laboratory data at initiation of } \\
\text { pomalidomide treatment }\end{array}$} \\
\hline eGFR $\left(\mathrm{ml} / \mathrm{min} / 1.73 \mathrm{~m}^{2}\right)$ & $64.2[29.1-99.2]$ & $57.1[13.9-101.3]$ & $64.6[42.7-81.4]$ & 0.93 \\
\hline WBC (per $\mu 1)$ & $3,955[2,980-4,930]$ & $4,950[2,830-7,040]$ & $3,420[2,300-8,380]$ & 0.74 \\
\hline Neutrophils (per $\mu 1$ ) & $2,531[1,788-3,352]$ & $2,871[1,330-5,914]$ & $2,155[1,035-5,698]$ & 0.79 \\
\hline Hemoglobin $(\mathrm{g} / \mu \mathrm{l})$ & 9.5 [7.6-11.4] & $9.2[6.9-11.9]$ & $9.5[8.8-10.6]$ & 0.99 \\
\hline Platelets $\left(\times 10^{4}\right.$ per $\left.\mu \mathrm{l}\right)$ & $15.3[15.2-15.3]$ & $13.5[7.2-29.9]$ & $11.5[6.1-25.8]$ & 0.79 \\
\hline \multicolumn{5}{|l|}{ Pomalidomide treatment } \\
\hline Exposure to pomalidomide (months) & $12[9-12]$ & $4[1-14]$ & $3[1-11]$ & 0.12 \\
\hline Number of treatment cycles & 9 [5-13] & $3[1-13]$ & $3[1-12]$ & 0.39 \\
\hline Daily treatment dose $(\mathrm{mg} /$ day $)(\mathrm{n}=68)$ & $4[2-4]$ & $3[3-4]$ & $3[1-4]$ & $<0.01$ \\
\hline Initiation treatment dose (mg/day) $(\mathrm{n}=14)$ & $4[4-4]$ & $4[3-4]$ & $4[2-4]$ & 0.26 \\
\hline Dose reduction of pomalidomide & $1(50.0)$ & $2(28.6)$ & $3(60.0)$ & 0.12 \\
\hline Dose interruption of pomalidomide & $2(100.0)$ & $2(28.6)$ & $3(60.0)$ & 0.12 \\
\hline Overall discontinuation of pomalidomide & $1(50.0)$ & $2(28.6)$ & $1(20.0)$ & 0.74 \\
\hline
\end{tabular}

ECOG, eastern cooperative oncology group; eGFR, estimated glomerular filtration rate; WBC, white blood cell. Data are presented as n (\%) or median [range].

Table IV. Adverse events associated with pomalidomide $(\mathrm{n}=14)$.

\begin{tabular}{lrrrr}
\hline & \multicolumn{2}{c}{ Grade 3/4 } & & All grades \\
\cline { 2 - 5 } Adverse events & No. & $(\%)$ & No. & $(\%)$ \\
\hline Hematological & & & & $(64.3)$ \\
$\quad$ Neutropenia & 9 & $(64.3)$ & 9 & $(78.6)$ \\
Anemia & 9 & $(64.3)$ & 11 & $(64.3)$ \\
Thrombocytopenia & 8 & $(57.1)$ & 9 & $(64.3)$ \\
Leukopenia & 8 & $(57.1)$ & 9 & $(7.1)$ \\
Febrile neutropenia & 1 & $(7.1)$ & & $(42.9)$ \\
Non-hematological & & & & $(14.3)$ \\
Infection & 6 & $(42.9)$ & 2 & $(42.9)$ \\
Pneumonia & 2 & $(14.3)$ & 7 & $(50.0)$ \\
Pyrexia & 0 & $(28.6)$ & 2 & $(14.3)$ \\
Fatigue & 4 & $(0.0)$ & 5 & $(35.7)$ \\
Peripheral neuropathy & 0 & $(0.0)$ & 4 & $(28.6)$ \\
Skin rash & 0 & $(0.0)$ & 1 & $(7.1)$ \\
Pruritus & 0 & $(0.0)$ & 4 & $(28.6)$ \\
Edema face & 0 & $(7.1)$ & 5 & $(35.7)$ \\
Increased serum ALT & 1 & $(0.0)$ & 3 & $(28.6)$ \\
Increased serum AST & 0 & $(0.0)$ & 4 & \\
Anorexia & 0 & $(7.1)$ & & \\
Constipation & 1 & & & \\
\hline
\end{tabular}

ALT, alanine aminotransferase; AST, aspartate aminotransferase. 
Table V. Reasons for dose reduction or interruption of pomalidomide treatment.

\begin{tabular}{|c|c|c|c|c|}
\hline \multirow{2}{*}{$\begin{array}{l}\text { Reasons for modification } \\
\text { Dose reduction }\end{array}$} & \multicolumn{2}{|c|}{ Patients $(\mathrm{n}=14), \mathrm{n}(\%)$} & \multicolumn{2}{|c|}{ Occasions $(\mathrm{n}=68), \mathrm{n}(\%)$} \\
\hline & 7 & $(50.0)$ & 11 & $(16.2)$ \\
\hline Neutropenia & 5 & $(35.7)$ & 8 & $(11.8)$ \\
\hline Thrombocytopenia & 2 & $(14.3)$ & 4 & $(5.9)$ \\
\hline Fatigue & 1 & $(7.1)$ & 1 & $(1.5)$ \\
\hline Infection & 0 & $(0.0)$ & 0 & $(0.0)$ \\
\hline Pneumonia & 0 & $(0.0)$ & 0 & $(0.0)$ \\
\hline Skin rash & 0 & $(0.0)$ & 0 & $(0.0)$ \\
\hline Increased serum ALT or AST & 2 & $(14.3)$ & 2 & $(2.9)$ \\
\hline Dose interruption & 7 & $(50.0)$ & 15 & $(22.1)$ \\
\hline Neutropenia & 5 & $(35.7)$ & 6 & $(8.8)$ \\
\hline Thrombocytopenia & 2 & $(14.3)$ & 2 & $(2.9)$ \\
\hline Fatigue & 3 & $(21.4)$ & 3 & $(4.4)$ \\
\hline Infection & 1 & $(7.1)$ & 0 & $(0.0)$ \\
\hline Pneumonia & 1 & $(7.1)$ & 1 & $(1.5)$ \\
\hline Skin rash & 1 & $(7.1)$ & 1 & $(1.5)$ \\
\hline Increased serum ALT or AST & 2 & $(14.3)$ & 2 & $(2.9)$ \\
\hline Overall discontinuation due to AEs & 4 & $(28.6)$ & & \\
\hline
\end{tabular}

AEs, adverse events; ALT, alanine aminotransferase; AST, aspartate aminotransferase.

POM. The tolerability of POM is generally low in patients with poor PS. Thus, aggressive treatment introduction should be avoided. There is a wide range of patients who may be treated by POM in the real-world setting, including patients with a high age or in a poor condition. It is important to determine tolerability and safety. In the present study, the patients who were unable to continue long-term treatment were switched to treatments including carfilzomib, daratumumab or panobinostat. The therapeutic options for patients with late-stage disease continue to expand, with continuous introduction of new treatments, including elotuzumab and ixazomib. These new agents have further prolonged the OS of RRMM patients. The most notable advantage of IMiDs is that they may be administered orally. This route of administration is convenient and particularly beneficial for older patients. Therefore, it is important to select treatment for RRMM patients taking into consideration patient convenience and treatment cost $(18,19)$.

In the present study, the incidence of grade 3/4 hematological AEs was compared with that of the MM-010 trial for neutropenia (64.3 vs. 49.7\%), anemia (78.6 vs. 33.0\%) and thrombocytopenia (64.3 vs. 24.1\%) (9). Compared with the Japanese clinical trial (MM-011), the incidence of neutropenia was comparable (64.3 vs. 63.8\%). However, anemia (78.6 vs. $41.7 \%$ ) and thrombocytopenia (64.3 vs. $31.4 \%$ ) were more frequent in the present study (20). Except for 3 patients who received only 1 treatment cycle, POM dose reduction or interruption occurred in $63.6 \%(7 / 11)$ of the patients. Therefore, we should confirm the incidence of hematological AEs carefully in a real-world setting.

LEN requires dose modification according to renal function. However, POM is indicated and is efficacious and safe even in patients with severe renal impairment (21). Maciocia et al (13) reported no significant difference in response, survival, or tolerability by renal function. Therefore,
POM is easier to use compared with LEN for patients with renal impairment. However, POM may only be used in patients who have received at least 2 prior therapies, including LEN and BOR. This affects the timing of treatment initiation due to patient restrictions. It may also be necessary to consider introducing POM in early myeloma treatment.

The capsule dosing of POM is convenient for patients. There are 2 types of capsules ( 2.5 and $5 \mathrm{mg}$ ) in LEN. The usual dose of LEN is $25 \mathrm{mg}$ for MM patients; therefore, the patient must take 5 capsules, which may be inconvenient. By contrast, POM is available in 4 doses $(1,2,3$ and $4 \mathrm{mg})$. Therefore, patients must only take 1 capsule, which may contribute to improved compliance due to the convenience. However, when POM dose reduction is required, it is necessary to change to a different capsule. If the patients have not completed one cycle of treatment, they are not able to take the remaining POM. With regard to inventory, it is difficult to continuously stock all 4 expensive dose types of POM in the hospital or pharmacy, and the expiration date of POM is only 4 years. As we previously experienced having to discard expired POM in our hospital, Thus, the patient condition must be carefully determined and a close communication with the prescribing physician must be maintained.

In conclusion, we herein conducted a retrospective analysis of RRMM patients treated with POM to describe its tolerability and safety in a real-world clinical setting. The age of the patients receiving POM was higher compared with that reported in the clinical trials. The incidence of grade 3/4 hematological AEs was high and the tolerability of POM was low for patients with poor PS. The most frequent treatment dose was $3 \mathrm{mg} /$ day. POM dosage should be reduced in the early phases, but POM may be administered to frail patients in a real-world setting. It is crucial that we pay close attention to elderly or poor PS patients in particular. In that manner, we 
may expect an expansion of the treatment options available to RRMM patients by assessing the tolerability and safety of POM. Our real-world experience may provide confirmatory support for further research on POM. However, the number of patients included in this study was small, real-world data from multiple centers must be accumulated to confirm our results.

\section{Acknowledgements}

Not applicable.

\section{Funding}

No funding was received.

\section{Availability of data and materials}

The datasets used and analyzed during the present study are available from the corresponding author on reasonable request.

\section{Authors' contributions}

EU and MK designed the study and prepared the manuscript. EU and ST observed the patients' condition and collected clinical data. EU and MI analyzed the data. HT and TY interpreted the data. All authors have read and approved the final version of the manuscript.

\section{Ethics approval and consent to participate}

This study was performed according to the principles set out in the 1964 Declaration of Helsinki and all subsequent revisions, and was approved by the Ethics Committee at Ogaki Municipal Hospital (20180628-6).

\section{Patient consent for publication}

Not applicable.

\section{Competing interests}

The authors declare that they have no competing interests.

\section{References}

1. Kyle RA, Gertz MA, Witzig TE, Lust JA, Lacy MQ, Dispenzieri A, Fonseca R, Rajkumar SV, Offord JR, Larson DR, et al: Review of 1027 patients with newly diagnosed multiple myeloma. Mayo Clin Proc 78: 21-33, 2003.

2. Kumar SK, Dispenzieri A, Lacy MQ, Gertz MA, Buadi FK, Pandey S, Kapoor P, Dingli D, Hayman SR, Leung N, et al: Continued improvement in survival in multiple myeloma: Changes in early mortality and outcomes in older patients. Leukemia 28: 1122-1128, 2014.

3. Dimopoulos MA, Chen C, Spencer A, Niesvizky R, Attal M, Stadtmauer EA, Petrucci MT, Yu Z, Olesnyckyj M, Zeldis JB, et al: Long-term follow-up on overall survival from the MM-009 and MM-010 phase III trials of lenalidomide plus dexamethasone in patients with relapsed or refractory multiple myeloma. Leukemia 23: 2147-2152, 2009.

4. Kumar SK, Lee JH, Lahuerta JJ, Morgan G, Richardson PG, Crowley J, Haessler J, Feather J, Hoering A, Moreau P, et al: Risk of progression and survival in multiple myeloma relapsing after therapy with IMiDs and bortezomib: A multicenter international myeloma working group study. Leukemia 26: 149-157, 2012.
5. Nijhof IS, van de Donk NWCJ, Zweegman S and Lokhorst HM: Current and new therapeutic strategies for relapsed and refractory multiple myeloma: An update. Drugs 78: 19-37, 2018.

6. Ocio EM, Fernández-Lázaro D, San-Segundo L, López-Corral L, Corchete LA, Gutiérrez NC, Garayoa M, Paíno T, García-Gómez A, Delgado M, et al: In vivo murine model of acquired resistance in myeloma reveals differential mechanisms for lenalidomide and pomalidomide in combination with dexamethasone. Leukemia 29: 705-714, 2015.

7. Miguel JS, Weisel K, Moreau P, Lacy M, Song K, Delforge M, Karlin L, Goldschmidt H, Banos A, Oriol A, et al: Pomalidomide plus low-dose dexamethasone versus high-dose dexamethasone alone for patients with relapsed and refractory multiple myeloma (MM-003): A randomised, open-label, phase 3 trial. Lancet Oncol 14: 1055-1066, 2013.

8. Richardson PG, Siegel DS, Vij R, Hofmeister CC, Baz R, Jagannath S, Chen C, Lonial S, Jakubowiak A, Bahlis N, et al: Pomalidomide alone or in combination with low-dose dexamethasone in relapsed and refractory multiple myeloma: A randomized phase 2 study. Blood 20: 1826-1832, 2014.

9. Dimopoulos MA, Palumbo A, Corradini P, Cavo M, Delforge M, Di Raimondo F, Weisel KC, Oriol A, Hansson M, Vacca A, et al: Safety and efficacy of pomalidomide plus low-dose dexamethasone in STRATUS (MM-010): A phase 3b study in refractory multiple myeloma. Blood 128: 497-503, 2016.

10. Moreau P, Dimopoulos MA, Richardson PG, Siegel DS, Cavo M, Corradini P, Weisel K, Delforge M, O'Gorman P, Song K, et al: Adverse event management in patients with relapsed and refractory multiple myeloma taking pomalidomide plus low-dose dexamethasone: A pooled analysis. Eur J Haematol 99: 199-206, 2017.

11. US Department Of Health And Human Services: Common terminology criteria for adverse events (CTCAE) version 4.0. United States, National Cancer Institute, 2009.

12. Sriskandarajah P, Pawlyn C, Mohammed K, Dearden CE, Davies FE, Morgan GJ, Boyd KD and Kaiser MF: The efficacy and tolerability of pomalidomide in relapsed/refractory myeloma patients in a 'real-world' study: The Royal Marsden Hospital experience. Leuk Lymphoma 58: 494-497, 2017.

13. Maciocia N, Melville A, Cheesman S, Sharpley F, Ramasamy K, Streetly M, Jenner M, Benjamin R, Schey S, Maciocia P, et al: Real-world use of pomalidomide and dexamethasone in double refractory multiple myeloma suggests benefit in renal impairment and adverse genetics: A multi-centre UK experience. Br J Haematol 176: 908-917, 2017.

14. Ailawadhi S, Mikhael JR, LaPlant BR, Laumann KM, Kumar S, Roy V, Dingli D, Bergsagel PL, Buadi FK, Rajkumar SV, et al: Pomalidomide-dexamethasone in refractory multiple myeloma: Long-term follow-up of a multi-cohort phase II clinical trial. Leukemia 32: 719-728, 2018.

15. Fouquet G, Pegourie B, Macro M, Petillon MO, Karlin L, Caillot D, Roussel M, Arnulf B, Mathiot C, Marit G, et al: Safe and prolonged survival with long-term exposure to pomalidomide in relapsed/refractory myeloma. Ann Oncol 27: 902-907, 2016.

16. Gueneau P, Chretien ML, Cransac-Miet A, Lafon I, Favennec C, Guy J, Caillot D and Boulin M: Efficacy, safety, and cost of pomalidomide in relapsed and refractory multiple myeloma. Eur J Haematol 100: 518-525, 2018.

17. Palmas A, Piras G, Uras A, Asproni R, Murineddu M, Monne M, Stradoni R and Latte G: Pomalidomide in heavily pretreated refractory multiple myeloma: A case report. Future Oncol 13: 7-9, 2017.

18. Chen CC, Parikh K, Abouzaid S, Purnomo L, McGuiness CB, Hussein M and Wade RL: Real-world treatment patterns, time to next treatment, and economic outcomes in relapsed or refractory multiple myeloma patients treated with pomalidomide or carfilzomib. J Manag Care Spec Pharm 23: 236-246, 2017.

19. Pelligra CG, Parikh K, Guo S, Chandler C, Mouro J, Abouzaid S and Ailawadhi S: Cost-effectiveness of pomalidomide, carfilzomib, and daratumumab for the treatment of patients with heavily pretreated relapsed-refractory multiple myeloma in the United States. Clin Ther 39: 1986-2005.e5, 2017.

20. Ichinohe T, Kuroda Y, Okamoto S, Matsue K, Iida S, Sunami K, Komeno T, Suzuki K, Ando K, Taniwaki M, et al: A multicenter phase 2 study of pomalidomide plus dexamethasone in patients with relapsed and refractory multiple myeloma: The Japanese MM-011 trial. Exp Hematol Oncol 5: 11, 2016.

21. Dimopoulos M, Weisel K, van de Donk NWCJ, Ramasamy K, Gamberi B, Streetly M, Offidani M, Bridoux F, de la Rubia J, Mateos MV, et al: Pomalidomide plus low-dose dexamethasone in patients with relapsed/refractory multiple myeloma and renal impairment: Results from a phase II trial. J Clin Oncol 36: 2035-2043, 2018. 